\title{
On Special Berwald Metrics
}

\author{
Akbar TAYEBI ${ }^{\dagger}$ and Esmaeil PEYGHAN ${ }^{\ddagger}$ \\ $\dagger$ Department of Mathematics, Faculty of Science, Qom University, Qom, Iran \\ E-mail: akbar.tayebi@gmail.com \\ $\ddagger$ Department of Mathematics, Faculty of Science, Arak University, Arak, Iran \\ E-mail: epeyghan@gmail.com,e-peyghan@araku.ac.ir
}

Received November 01, 2009, in final form January 17, 2010; Published online January 20, 2010 doi:10.3842/SIGMA.2010.008

\begin{abstract}
In this paper, we study a class of Finsler metrics which contains the class of Berwald metrics as a special case. We prove that every Finsler metric in this class is a generalized Douglas-Weyl metric. Then we study isotropic flag curvature Finsler metrics in this class. Finally we show that on this class of Finsler metrics, the notion of Landsberg and weakly Landsberg curvature are equivalent.
\end{abstract}

Key words: Randers metric; Douglas curvature; Berwald curvature

2010 Mathematics Subject Classification: 53C60; 53C25

\section{Introduction}

For a Finsler metric $F=F(x, y)$, its geodesics curves are characterized by the system of differential equations $\ddot{c}^{i}+2 G^{i}(\dot{c})=0$, where the local functions $G^{i}=G^{i}(x, y)$ are called the spray coefficients. A Finsler metric $F$ is called a Berwald metric if $G^{i}=\frac{1}{2} \Gamma_{j k}^{i}(x) y^{j} y^{k}$ is quadratic in $y \in T_{x} M$ for any $x \in M$. It is proved that on a Berwald space, the parallel translation along any geodesic preserves the Minkowski functionals [7]. Thus Berwald spaces can be viewed as Finsler spaces modeled on a single Minkowski space.

Recently by using the structure of Funk metric, Chen-Shen introduce the notion of isotropic Berwald metrics $[6,16]$. This motivates us to study special forms of Berwald metrics.

Let $(M, F)$ be a two-dimensional Finsler manifold. We refer to the Berwald's frame $\left(\ell^{i}, m^{i}\right)$ where $\ell^{i}=y^{i} / F(y), m^{i}$ is the unit vector with $\ell_{i} m^{i}=0, \ell_{i}=g_{i j} \ell^{i}$ and $g_{i j}$ is the fundamental tensor of Finsler metric $F$. Then the Berwald curvature is given by

$$
B^{i}{ }_{j k l}=F^{-1}\left(-2 I_{, 1} \ell^{i}+I_{2} m^{i}\right) m_{j} m_{k} m_{l},
$$

where $I$ is 0 -homogeneous function called the main scalar of Finsler metric and $I_{2}=I_{, 2}+I_{, 1 \mid 2}$ (see [2, page 689]). By (1), we have

$$
B_{j k l}^{i}=-\frac{2 I_{, 1}}{3 F^{2}}\left(m_{j} h_{k l}+m_{k} h_{j l}+m_{l} h_{j k}\right) y^{i}+\frac{I_{2}}{3 F}\left(h_{j}^{i} h_{k l}+h_{k}^{i} h_{j l}+h_{l}^{i} h_{j k}\right),
$$

where $h_{i j}:=m_{i} m_{j}$ is called the angular metric. Using the special form of Berwald curvature for Finsler surfaces, we define a new class of Finsler metrics on $n$-dimensional Finsler manifolds which their Berwald curvature satisfy in following

$$
B^{i}{ }_{j k l}=\left(\mu_{j} h_{k l}+\mu_{k} h_{j l}+\mu_{l} h_{j k}\right) y^{i}+\lambda\left(h_{j}^{i} h_{k l}+h_{k}^{i} h_{j l}+h_{l}^{i} h_{j k}\right)
$$

where $\mu_{i}=\mu_{i}(x, y)$ and $\lambda=\lambda(x, y)$ are homogeneous functions of degrees -2 and -1 with respect to $y$, respectively. By definition of Berwald curvature, the function $\mu_{i}$ satisfies $\mu_{i} y^{i}=0$ [12]. 
The Douglas tensor is another non-Riemanian curvature defined as follows

$$
D_{j k l}^{i}:=\left(G^{i}-\frac{1}{n+1} \frac{\partial G^{m}}{\partial y^{m}} y^{i}\right)_{y^{j} y^{k} y^{l}} .
$$

Douglas curvature is a non-Riemannian projective invariant constructed from the Berwald curvature. The notion of Douglas curvature was proposed by Bácsó and Matsumoto as a generalization of Berwald curvature [4]. We show that a Finsler metric satisfies (2) with vanishing Douglas tensor is a Randers metric (see Proposition 1). A Finsler metric is called a generalized DouglasWeyl (GDW) metric if the Douglas tensor satisfy in $h_{\alpha}^{i} D_{j k l \mid m}^{\alpha} y^{m}=0$ [10]. In [5], Bácsó-Papp show that this class of Finsler metrics is closed under projective transformation. We prove that a Finsler metric satisfies (2) is a GDW-metric.

Theorem 1. Every Finsler metric satisfying (2) is a GDW-metric.

Theorem 1, shows that every two-dimensional Finsler metric is a generalized Douglas-Weyl metric.

For a Finsler manifold $(M, F)$, the flag curvature is a function $\mathbf{K}(P, y)$ of tangent planes $P \subset T_{x} M$ and directions $y \in P . F$ is said to be of isotropic flag curvature if $\mathbf{K}(P, y)=\mathbf{K}(x)$ and constant flag curvature if $\mathbf{K}(P, y)=$ const.

Theorem 2. Let $F$ be a Finsler metric of non-zero isotropic flag curvature $\mathbf{K}=\mathbf{K}(x)$ on a manifold $M$. Suppose that $F$ satisfies (2). Then $F$ is a Riemannian metric if and only if $\mu_{i}$ is constant along geodesics.

Beside the Berwald curvature, there are several important Finslerian curvature. Let $(M, F)$ be a Finsler manifold. The second derivatives of $\frac{1}{2} F_{x}^{2}$ at $y \in T_{x} M_{0}$ is an inner product $\mathbf{g}_{y}$ on $T_{x} M$. The third order derivatives of $\frac{1}{2} F_{x}^{2}$ at $y \in T_{x} M_{0}$ is a symmetric trilinear forms $\mathbf{C}_{y}$ on $T_{x} M$. We call $\mathbf{g}_{y}$ and $\mathbf{C}_{y}$ the fundamental form and the Cartan torsion, respectively. The rate of change of the Cartan torsion along geodesics is the Landsberg curvature $\mathbf{L}_{y}$ on $T_{x} M$

for any $y \in T_{x} M_{0}$. Set $\mathbf{J}_{y}:=\sum_{i=1}^{n} \mathbf{L}_{y}\left(e_{i}, e_{i}, \cdot\right)$, where $\left\{e_{i}\right\}$ is an orthonormal basis for $\left(T_{x} M, g_{y}\right)$. $\mathbf{J}_{y}$ is called the mean Landsberg curvature. $F$ is said to be Landsbergian if $\mathbf{L}=0$, and weakly Landsbergian if $\mathbf{J}=0[13,14]$.

In this paper, we prove that on Finsler manifolds satisfies (2), the notions of Landsberg and weakly Landsberg metric are equivalent.

Theorem 3. Let $(M, F)$ be a Finsler manifold satisfying (2). Then $\mathbf{L}=0$ if and only if $\mathbf{J}=0$.

There are many connections in Finsler geometry [15]. In this paper, we use the Berwald connection and the $h$ - and $v$-covariant derivatives of a Finsler tensor field are denoted by "|" and "," respectively.

\section{Preliminaries}

Let $M$ be a n-dimensional $C^{\infty}$ manifold. Denote by $T_{x} M$ the tangent space at $x \in M$, by $T M=\cup_{x \in M} T_{x} M$ the tangent bundle of $M$, and by $T M_{0}=T M \backslash\{0\}$ the slit tangent bundle on $M$. A Finsler metric on $M$ is a function $F: T M \rightarrow[0, \infty)$ which has the following properties: (i) $F$ is $C^{\infty}$ on $T M_{0}$; $(i i) F$ is positively 1-homogeneous on the fibers of tangent bundle $T M$, and (iii) for each $y \in T_{x} M$, the following quadratic form $g_{y}$ on $T_{x} M$ is positive definite,

$$
g_{y}(u, v):=\left.\frac{1}{2} \frac{d^{2}}{d s d t}\left[F^{2}(y+s u+t v)\right]\right|_{s, t=0}, \quad u, v \in T_{x} M .
$$


Let $x \in M$ and $F_{x}:=\left.F\right|_{T_{x} M}$. To measure the non-Euclidean feature of $F_{x}$, define $\mathbf{C}_{y}: T_{x} M \times$ $T_{x} M \times T_{x} M \rightarrow \mathbb{R}$ by

$$
\mathbf{C}_{y}(u, v, w):=\left.\frac{1}{2} \frac{d}{d t}\left[g_{y+t w}(u, v)\right]\right|_{t=0}, \quad u, v, w \in T_{x} M .
$$

The family $\mathbf{C}:=\left\{\mathbf{C}_{\mathbf{y}}\right\}_{\mathbf{y} \in \mathbf{T} \mathbf{M}_{\mathbf{0}}}$ is called the Cartan torsion. It is well known that $\mathbf{C}=0$ if and only if $F$ is Riemannian [14]. For $y \in T_{x} M_{0}$, define mean Cartan torsion $\mathbf{I}_{y}$ by $\mathbf{I}_{y}(u):=I_{i}(y) u^{i}$, where $I_{i}:=g^{j k} C_{i j k}, g^{j k}$ is the inverse of $g_{j k}$ and $u=\left.u^{i} \frac{\partial}{\partial x^{i}}\right|_{x}$. By Deicke's theorem, $F$ is Riemannian if and only if $\mathbf{I}_{y}=0$ [13].

Let $\alpha=\sqrt{a_{i j}(x) y^{i} y^{j}}$ be a Riemannian metric, and $\beta=b_{i}(x) y^{i}$ be a 1-form on $M$ with $b=\sqrt{a^{i j} b_{i} b_{j}}<1$. The Finsler metric $F=\alpha+\beta$ is called a Randers metric.

Let $(M, F)$ be a Finsler manifold. Then for a non-zero vector $y \in T_{x} M_{0}$, define the Matsumoto torsion $\mathbf{M}_{y}: T_{x} M \otimes T_{x} M \otimes T_{x} M \rightarrow \mathbb{R}$ by $\mathbf{M}_{y}(u, v, w):=M_{i j k}(y) u^{i} v^{j} w^{k}$ where

$$
M_{i j k}:=C_{i j k}-\frac{1}{n+1}\left\{I_{i} h_{j k}+I_{j} h_{i k}+I_{k} h_{i j}\right\}
$$

$h_{i j}:=F F_{y^{i} y^{j}}=g_{i j}-\frac{1}{F^{2}} g_{i p} y^{p} g_{j q} y^{q}$ is the angular metric and $I_{i}:=g^{j k} C_{i j k}$ is the mean Cartan torsion. By definition, we have $h_{i j} y^{i}=0, h_{j}^{i}=\delta_{j}^{i}-F^{-2} y^{i} y_{j}, y_{j}=g_{i j} y^{i}, h_{j}^{i} h_{i k}=h_{j k}$ and $h_{i}^{i}=n-1$. A Finsler metric $F$ is said to be $C$-reducible if $\mathbf{M}_{y}=0$. This quantity is introduced by Matsumoto [8]. Matsumoto proves that every Randers metric satisfies that $\mathbf{M}_{y}=0$. Later on, Matsumoto-Hōjō proves that the converse is true too.

Lemma 1 ([9]). A Finsler metric $F$ on a manifold of dimension $n \geq 3$ is a Randers metric if and only if $\mathbf{M}_{y}=0, \forall y \in T M_{0}$.

Let us consider the pull-back tangent bundle $\pi^{*} T M$ over $T M_{0}$ defined by

$$
\pi^{*} T M=\left\{(u, v) \in T M_{0} \times T M_{0} \mid \pi(u)=\pi(v)\right\} .
$$

Let $\nabla$ be the Berwald connection. Let $\left\{e_{i}\right\}_{i=1}^{n}$ be a local orthonormal (with respect to $g$ ) frame field for the pulled-back bundle $\pi^{*} T M$ such that $e_{n}=\ell$, where $\ell$ is the canonical section of $\pi^{*} T M$ defined by $\ell_{y}=y / F(y)$. Let $\left\{\omega^{i}\right\}_{i=1}^{n}$ be its dual co-frame field. Put $\nabla e_{i}=\omega^{j}{ }_{i} \otimes e_{j}$, where $\left\{\omega^{j}{ }_{i}\right\}$ is called the connection forms of $\nabla$ with respect to $\left\{e_{i}\right\}$. Put $\omega^{n+i}:=\omega^{i}{ }_{n}+d(\log F) \delta_{n}^{i}$. It is easy to show that $\left\{\omega^{i}, \omega^{n+i}\right\}_{i=1}^{n}$ is a local basis for $T^{*}\left(T M_{0}\right)$. Since $\left\{\Omega_{i}^{j}\right\}$ are 2 -forms on $T M_{0}$, they can be expanded as

$$
\Omega_{i}^{j}=\frac{1}{2} R_{i k l}^{j} \omega^{k} \wedge \omega^{l}+B_{i k l}^{j} \omega^{k} \wedge \omega^{n+l} .
$$

Let $\left\{\bar{e}_{i}, \dot{e}_{i}\right\}_{i=1}^{n}$ be the local basis for $T\left(T M_{0}\right)$, which is dual to $\left\{\omega^{i}, \omega^{n+i}\right\}_{i=1}^{n}$. The objects $R$ and $B$ are called, respectively, the $h h$ - and $h v$-curvature tensors of the Berwald connection with the components $R\left(\bar{e}_{k}, \bar{e}_{l}\right) e_{i}=R_{i k l}^{j} e_{j}$ and $P\left(\bar{e}_{k}, \dot{e}_{l}\right) e_{i}=P_{i k l}^{j} e_{j}$ [15]. With the Berwald connection, we define covariant derivatives of quantities on $T M_{0}$ in the usual way. For example, for a scalar function $f$, we define $f_{\mid i}$ and $f_{\cdot i}$ by

$$
d f=f_{\mid i} \omega^{i}+f_{, i} \omega^{n+i},
$$

where "|" and "," denote the $h$ - and $v$-covariant derivatives, respectively.

The horizontal covariant derivatives of $\mathbf{C}$ along geodesics give rise to the Landsberg curvature $\mathbf{L}_{y}: T_{x} M \times T_{x} M \times T_{x} M \rightarrow \mathbb{R}$ defined by

$$
\mathbf{L}_{y}(u, v, w):=L_{i j k}(y) u^{i} v^{j} w^{k},
$$

where $L_{i j k}:=C_{i j k \mid s} y^{s}, u=\left.u^{i} \frac{\partial}{\partial x^{i}}\right|_{x}, v=\left.v^{i} \frac{\partial}{\partial x^{i}}\right|_{x}$ and $w=\left.w^{i} \frac{\partial}{\partial x^{i}}\right|_{x}$. The family $\mathbf{L}:=\left\{\mathbf{L}_{y}\right\}_{y \in T M_{0}}$ is called the Landsberg curvature. A Finsler metric is called a Landsberg metric if $\mathbf{L}=\mathbf{0}$. The 
horizontal covariant derivatives of $\mathbf{I}$ along geodesics give rise to the mean Landsberg curvature $\mathbf{J}_{y}(u):=J_{i}(y) u^{i}$, where $J_{i}:=g^{j k} L_{i j k}$. A Finsler metric is said to be weakly Landsbergian if $\mathbf{J}=0$.

Given a Finsler manifold $(M, F)$, then a global vector field $G$ is induced by $F$ on $T M_{0}$, which in a standard coordinate $\left(x^{i}, y^{i}\right)$ for $T M_{0}$ is given by

$$
G=y^{i} \frac{\partial}{\partial x^{i}}-2 G^{i}(x, y) \frac{\partial}{\partial y^{i}},
$$

where $G^{i}(y)$ are local functions on $T M$ given by

$$
G^{i}(y):=\frac{1}{4} g^{i l}(y)\left\{\frac{\partial^{2}\left[F^{2}\right]}{\partial x^{k} \partial y^{l}}(y) y^{k}-\frac{\partial\left[F^{2}\right]}{\partial x^{l}}(y)\right\}, \quad y \in T_{x} M .
$$

$G$ is called the spray associated to $(M, F)$. In local coordinates, a curve $c(t)$ is a geodesic if and only if its coordinates $\left(c^{i}(t)\right)$ satisfy $\ddot{c}^{i}+2 G^{i}(\dot{c})=0$.

For a tangent vector $y \in T_{x} M_{0}$, define $\mathbf{B}_{y}: T_{x} M \otimes T_{x} M \otimes T_{x} M \rightarrow T_{x} M$ and $\mathbf{E}_{y}: T_{x} M \otimes$ $T_{x} M \rightarrow \mathbb{R}$ by $\mathbf{B}_{y}(u, v, w):=\left.B^{i}{ }_{j k l}(y) u^{j} v^{k} w^{l} \frac{\partial}{\partial x^{i}}\right|_{x}$ and $\mathbf{E}_{y}(u, v):=E_{j k}(y) u^{j} v^{k}$ where

$$
B_{j k l}^{i}(y):=\frac{\partial^{3} G^{i}}{\partial y^{j} \partial y^{k} \partial y^{l}}(y), \quad E_{j k}(y):=\frac{1}{2} B_{j k m}^{m}(y) .
$$

$\mathbf{B}$ and $\mathbf{E}$ are called the Berwald curvature and mean Berwald curvature, respectively. Then $F$ is called a Berwald metric and weakly Berwald metric if $\mathbf{B}=\mathbf{0}$ and $\mathbf{E}=\mathbf{0}$, respectively [14]. By definition of Berwald and mean Berwald curvatures, we have

$$
y^{j} B^{i}{ }_{j k l}=y^{k} B^{i}{ }_{j k l}=y^{l} B^{i}{ }_{j k l}=0, \quad y^{j} E_{j k}=y^{k} E_{j k}=0 .
$$

The Riemann curvature $\mathbf{R}_{y}=\left.R_{k}^{i} d x^{k} \otimes \frac{\partial}{\partial x^{i}}\right|_{x}: T_{x} M \rightarrow T_{x} M$ is a family of linear maps on tangent spaces, defined by

$$
R_{k}^{i}=2 \frac{\partial G^{i}}{\partial x^{k}}-y^{j} \frac{\partial^{2} G^{i}}{\partial x^{j} \partial y^{k}}+2 G^{j} \frac{\partial^{2} G^{i}}{\partial y^{j} \partial y^{k}}-\frac{\partial G^{i}}{\partial y^{j}} \frac{\partial G^{j}}{\partial y^{k}} .
$$

The flag curvature in Finsler geometry is a natural extension of the sectional curvature in Riemannian geometry was first introduced by L. Berwald [3]. For a flag $P=\operatorname{span}\{y, u\} \subset T_{x} M$ with flagpole $y$, the flag curvature $\mathbf{K}=\mathbf{K}(P, y)$ is defined by

$$
\mathbf{K}(P, y):=\frac{\mathbf{g}_{y}\left(u, \mathbf{R}_{y}(u)\right)}{\mathbf{g}_{y}(y, y) \mathbf{g}_{y}(u, u)-\mathbf{g}_{y}(y, u)^{2}} .
$$

When $F$ is Riemannian, $\mathbf{K}=\mathbf{K}(P)$ is independent of $y \in P$, and is the sectional curvature of $P$. We say that a Finsler metric $F$ is of scalar curvature if for any $y \in T_{x} M$, the flag curvature $\mathbf{K}=\mathbf{K}(x, y)$ is a scalar function on the slit tangent bundle $T M_{0}$. If $\mathbf{K}=$ const, then $F$ is said to be of constant flag curvature. A Finsler metric $F$ is called isotropic flag curvature, if $\mathbf{K}=\mathbf{K}(x)$.

In [1], Akbar-Zadeh considered a non-Riemannian quantity $\mathbf{H}$ which is obtained from the mean Berwald curvature by the covariant horizontal differentiation along geodesics. This is a positively homogeneous scalar function of degree zero on the slit tangent bundle. The quantity $\mathbf{H}_{y}=H_{i j} d x^{i} \otimes d x^{j}$ is defined as the covariant derivative of $\mathbf{E}$ along geodesics [11]. More precisely

$$
H_{i j}:=E_{i j \mid m} y^{m} \text {. }
$$

In local coordinates, we have

$$
2 H_{i j}=y^{m} \frac{\partial^{4} G^{k}}{\partial y^{i} \partial y^{j} \partial y^{k} \partial x^{m}}-2 G^{m} \frac{\partial^{4} G^{k}}{\partial y^{i} \partial y^{j} \partial y^{k} \partial y^{m}}-\frac{\partial G^{m}}{\partial y^{i}} \frac{\partial^{3} G^{k}}{\partial y^{j} \partial y^{k} \partial y^{m}}-\frac{\partial G^{m}}{\partial y^{j}} \frac{\partial^{4} G^{k}}{\partial y^{i} \partial y^{k} \partial y^{m}} .
$$

Akbar-Zadeh proved the following:

Theorem 4 ([1]). Let $F$ be a Finsler metric of scalar curvature on an n-dimensional manifold $M(n \geq 3)$. Then the flag curvature $\mathbf{K}=$ const if and only if $\mathbf{H}=0$. 


\section{Proof of Theorem 1}

Lemma 2. Let $(M, F)$ be a Finsler manifold. Suppose that the Cartan tensor satisfies in $C_{i j k}=B_{i} h_{j k}+B_{j} h_{i k}+B_{k} h_{i j}$ with $y^{i} B_{i}=0$. Then $F$ is a $C$-reducible metric.

Proof. Suppose that the Cartan tensor of the Finsler metric $F$ satisfies in

$$
C_{i j k}=B_{i} h_{j k}+B_{j} h_{i k}+B_{k} h_{i j}
$$

Contracting (4) with $g^{i j}$ yields

$$
I_{k}=B_{i} h_{k}^{i}+B_{j} h_{k}^{j}+(n-1) B_{k} .
$$

Using (5) and $B_{i} h_{k}^{i}=B_{j} h_{k}^{j}=B_{k}$, we get $I_{i}=(n+1) B_{i}$. Putting this relation in (4), we conclude that $F$ is a $C$-reducible Finsler metric.

Lemma 3. Let $(M, F)$ be a Finsler metric. Then $F$ is a $G D W$-metric if and only if

$$
D^{i}{ }_{j k l \mid s} y^{s}=T_{j k l} y^{i}
$$

for some tensor $T_{j k l}$ on manifold $M$.

Proof. Let $F$ be is a GDW-metric

$$
h_{m}^{i} D_{j k l \mid s}^{m} y^{s}=0 .
$$

This yields

$$
D^{i}{ }_{j k l \mid s} y^{s}=\left(F^{-2} y_{m} D_{j k l \mid s}^{m}\right) y^{i} .
$$

Therefore $T_{j k l}:=F^{-2} y_{m} D_{j k l \mid s}^{m}$. The proof of converse is trivial.

Equation (6) is equivalent to the condition that, for any parallel vector fields $U=U(t)$, $V=V(t)$ and $W=W(t)$ along a geodesic $c(t)$, there is a function $T=T(t)$ such that

$$
\frac{d}{d t}\left[D_{\dot{c}}(U, V, W)\right]=T \dot{c} .
$$

The geometric meaning of the above identity is that the rate of change of the Douglas curvature along a geodesic is tangent to the geodesic.

Proposition 1. Let $(M, F)$ be a Finsler manifold satisfies (2) with dimension $n \geq 3$. Suppose that the Douglas tensor of $F$ vanishes. Then $F$ is a Randers metric.

Proof. Since $F$ satisfies (2), then by considering $\mu_{i} y^{i}=0$ we get

$$
2 E_{j k}=(n+1) \lambda h_{i j} .
$$

On the other hand, we have

$$
h_{i j, k}=2 C_{i j k}-F^{-2}\left(y_{j} h_{i k}+y_{i} h_{j k}\right),
$$

which implies that

$$
2 E_{j k, l}=(n+1) \lambda_{, l} h_{j k}+(n+1) \lambda\left\{2 C_{j k l}-F^{-2}\left(y_{k} h_{j l}+y_{j} h_{k l}\right)\right\} .
$$


Putting (2), (7) and (8) in (3) yields

$$
D^{i}{ }_{j k l}=\left\{\mu_{j} h_{k l}+\mu_{k} h_{j l}+\mu_{l} h_{j k}-2 \lambda C_{j k l}\right\} y^{i}-\left(\lambda y_{l} F^{-2}+\lambda_{, l}\right) h_{j k} y^{i} .
$$

For the Douglas curvature, we have $D^{i}{ }_{j k l}=D^{i}{ }_{j l k}$. Then by (9), we conclude that

$$
\lambda y_{l} F^{-2}+\lambda_{, l}=0
$$

From (9) and (10) we deduce

$$
D^{i}{ }_{j k l}=\left\{\mu_{j} h_{k l}+\mu_{k} h_{j l}+\mu_{l} h_{j k}-2 \lambda C_{j k l}\right\} y^{i} .
$$

Since $F$ is a Douglas metric, then

$$
C_{j k l}=\frac{1}{2 \lambda}\left\{\mu_{j} h_{k l}+\mu_{k} h_{j l}+\mu_{l} h_{j k}\right\} .
$$

By Lemmas 2 and 1, it follows that $F$ is a Randers metric.

Proof of Theorem 1. To prove the Theorem 1, we start with the equation (11):

$$
D^{i}{ }_{j k l}=\left\{\mu_{j} h_{k l}+\mu_{k} h_{j l}+\mu_{l} h_{j k}-2 \lambda C_{j k l}\right\} y^{i}
$$

Taking a horizontal derivation of (12) implies that

$$
D^{i}{ }_{j k l \mid s} y^{s}=\left\{\mu_{j}^{\prime} h_{k l}+\mu_{k}^{\prime} h_{j l}+\mu_{l}^{\prime} h_{j k}-2 \lambda^{\prime} C_{j k l}-2 \lambda L_{j k l}\right\} y^{i} .
$$

where $\lambda^{\prime}=\lambda_{\mid m} y^{m}$ and $\mu_{i}^{\prime}=\mu_{i \mid m} y^{m}$. By Lemma 3, $F$ is a GDW-metric with

$$
T_{j k l}=\mu_{j}^{\prime} h_{k l}+\mu_{k}^{\prime} h_{j l}+\mu_{l}^{\prime} h_{j k}-2 \lambda^{\prime} C_{j k l}-2 \lambda L_{j k l} .
$$

This completes the proof.

The Funk metric on a strongly convex domain $\mathbb{B}^{n} \subset \mathbb{R}^{n}$ is a non-negative function on $T \Omega=$ $\Omega \times \mathbb{R}^{n}$, which in the special case $\Omega=\mathbb{B}^{n}$ (the unit ball in the Euclidean space $\mathbb{R}^{n}$ ) is defined by the following explicit formula:

$$
F(y):=\frac{\sqrt{|y|^{2}-\left(|x|^{2}|y|^{2}-\langle x, y\rangle^{2}\right)}}{1-|x|^{2}}+\frac{\langle x, y\rangle}{1-|x|^{2}}, \quad y \in T_{x} \mathbb{B}^{n}=\mathbb{R}^{n}
$$

where $|\cdot|$ and $\langle\cdot, \cdot\rangle$ denote the Euclidean norm and inner product in $\mathbb{R}^{n}$, respectively [14]. The Funk metric on $\mathbb{B}^{n}$ is a Randers metric. The Berwald curvature of Funk metric is given by

$$
B_{j k l}^{i}=\frac{1}{2 F}\left\{h_{j}^{i} h_{k l}+h_{k}^{i} h_{j l}+h_{l}^{i} h_{j k}+2 C_{j k l} y^{i}\right\}
$$

Thus the Funk metric is a GDW-metric which does not satisfy (2). Then by Theorem 1, we conclude the following.

Corollary 1. The class of Finsler metrics satisfying (2) is a proper subset of the class of generalized Douglas-Weyl metrics. 


\section{Proof of Theorem 2}

To prove Theorem 2, we need the following.

Lemma $4([7,11])$. For the Berwald connection, the following Bianchi identities hold:

$$
\begin{aligned}
& R_{j k l \mid m}^{i}+R^{i}{ }_{j l m \mid k}+R^{i}{ }_{j m k \mid l}=0, \\
& B^{i}{ }_{j m l \mid k}-B^{i}{ }_{j k m \mid l}=R^{i}{ }_{j k l, m}, \\
& B_{j k l, m}^{i}=B^{i}{ }_{j k m, l} .
\end{aligned}
$$

Proof of Theorem 2. We have:

$$
R_{j k l}^{i}=\frac{1}{3}\left\{\frac{\partial^{2} R_{k}^{i}}{\partial y^{j} \partial y^{l}}-\frac{\partial^{2} R_{l}^{i}}{\partial y^{j} \partial y^{k}}\right\} .
$$

Here, we assume that a Finsler metric $F$ is of isotropic flag curvature $\mathbf{K}=\mathbf{K}(x)$. In local coordinates, $R_{k}^{i}=\mathbf{K}(x) F^{2} h_{k}^{i}$. Plugging this equation into (14) gives

$$
R^{i}{ }_{j k l}=\mathbf{K}\left\{g_{j l} \delta_{k}^{i}-g_{j k} \delta_{l}^{i}\right\} .
$$

Differentiating (15) with respect to $y^{m}$ gives a formula for $R^{i}{ }_{j k l, m}$ expressed in terms of $\mathbf{K}$ and its derivatives. Contracting (13) with $y^{k}$, we obtain

$$
B^{i}{ }_{j m l \mid k} y^{k}=2 \mathbf{K} C_{j m l} y^{i} .
$$

Multiplying (16) with $y_{i}$ implies that

$$
B^{i}{ }_{j m l \mid k} y^{k} y_{i}=2 \mathbf{K} F^{2} C_{j m l} \text {. }
$$

Since $F$ satisfies (2), then we have

$$
B^{i}{ }_{j k l \mid m} y^{m}=\left(\mu_{j}^{\prime} h_{k l}+\mu_{k}^{\prime} h_{j l}+\mu_{l}^{\prime} h_{j k}\right) y^{i}+\lambda^{\prime}\left(h_{j}^{i} h_{k l}+h_{k}^{i} h_{j l}+h_{l}^{i} h_{j k}\right) .
$$

By contracting (18) with $y_{i}$, we have

$$
B^{i}{ }_{j k l \mid m} y^{m} y_{i}=\left(\mu_{j}^{\prime} h_{k l}+\mu_{k}^{\prime} h_{j l}+\mu_{l}^{\prime} h_{j k}\right) F^{2} .
$$

By (17) and (19) we get

$$
\mu_{j}^{\prime} h_{k l}+\mu_{k}^{\prime} h_{j l}+\mu_{l}^{\prime} h_{j k}=2 \mathbf{K} C_{j k l} .
$$

Contracting with $g^{k l}$ yields

$$
\mu_{j}^{\prime}=\frac{2 \mathbf{K}}{n+1} I_{j}
$$

Since $\mathbf{K} \neq 0$, then by Deicke's theorem $F$ is a Riemannian metric if and only if $\mu_{j}^{\prime}=0$.

Theorem 5. Let $F$ be a Finsler metric on an n-dimensional manifold $M(n \geq 3)$ and satisfies (2). Suppose that $F$ is of scalar flag curvature $\mathbf{K}$. Then $\mathbf{K}=$ const if and only if $\lambda^{\prime}=0$.

Proof. Contracting $i$ and $l$ in (2) yields

$$
2 E_{j k}=(n+1) \lambda h_{j k} .
$$

By taking a horizontal derivative of this equation, we have

$$
2 H_{j k}=(n+1) \lambda^{\prime} h_{j k} .
$$

Therefore $H_{j k}=0$ if and only if $\lambda^{\prime}=0$. By Theorem 4, we get the proof. 


\section{$5 \quad$ Proof of Theorem 3}

In this section, we are going to prove Theorem 3.

Proof of Theorem 2. Let $F$ be a Finsler metric satisfy in following

$$
B^{i}{ }_{j k l}=\left(\mu_{j} h_{k l}+\mu_{k} h_{j l}+\mu_{l} h_{j k}\right) y^{i}+\lambda\left(h_{j}^{i} h_{k l}+h_{k}^{i} h_{j l}+h_{l}^{i} h_{j k}\right)
$$

where $\mu_{i}=\mu_{i}(x, y)$ and $\lambda=\lambda(x, y)$ are homogeneous functions of degrees -2 and -1 with respect to $y$, respectively. Contracting (20) with $y_{i}$ yields

$$
y_{i} B^{i}{ }_{j k l}=F^{2}\left(\mu_{j} h_{k l}+\mu_{k} h_{j l}+\mu_{l} h_{j k}\right)+\lambda y_{i}\left(h_{j}^{i} h_{k l}+h_{k}^{i} h_{j l}+h_{l}^{i} h_{j k}\right) .
$$

On the other hand, we have

$$
\begin{aligned}
& y_{i} B^{i}{ }_{j k l}=-2 L_{j k l}, \\
& y_{i} h_{m}^{i}=y_{i}\left(\delta_{m}^{i}-F^{-2} y^{i} y_{m}\right)=0 .
\end{aligned}
$$

See [14, page 84]. Using (21), (22) and (23), we get

$$
L_{j k l}=-\frac{1}{2} F^{2}\left\{\mu_{j} h_{k l}+\mu_{k} h_{j l}+\mu_{l} h_{j k}\right\} .
$$

By (24), it is obvious that if $\mu_{i}=0$ then $L_{j k l}=0$. Conversely let $F$ be a Landsberg metric. Then we have

$$
\mu_{j} h_{k l}+\mu_{k} h_{j l}+\mu_{l} h_{j k}=0 .
$$

Contracting (25) with $g^{k l}$ yields $\mu_{j}=0$. Then $F$ is a Landsberg metric if and only if $\mu_{j}=0$. Now, contracting (24) with $g^{k l}$ yields

$$
J_{j}=-\frac{1}{2}(n+1) F^{2} \mu_{j} .
$$

By (26), $J_{j}=0$ if and only if $\mu_{j}=0$. Then $\mathbf{L}=0$ if and only if $\mathbf{J}=0$.

By using the notion of Landsberg curvature, we define the stretch curvature $\boldsymbol{\Sigma}_{y}: T_{x} M \otimes$ $T_{x} M \otimes T_{x} M \otimes T_{x} M \rightarrow \mathbb{R}$ by $\boldsymbol{\Sigma}_{y}(u, v, w, z):=\Sigma_{i j k l}(y) u^{i} v^{j} w^{k} z^{l}$ where

$$
\Sigma_{i j k l}:=2\left(L_{i j k \mid l}-L_{i j l \mid k}\right) .
$$

In [3], L. Berwald has introduce the stretch curvature tensor $\boldsymbol{\Sigma}$ and showed that this tensor vanishes if and only if the length of a vector remains unchanged under the parallel displacement along an infinitesimal parallelogram.

Theorem 6. Let $(M, F)$ be a Finsler manifold on which (2) holds. Suppose that $F$ is a stretch metric. Then $\mu_{j}$ is constant along any Finslerian geodesics.

Proof. Taking a horizontal derivation of (24) yields

$$
L_{i j k \mid l}=-\frac{1}{2} F^{2}\left\{\mu_{i \mid l} h_{j k}+\mu_{j \mid l} h_{k i}+\mu_{k \mid l} h_{i j}\right\} .
$$

Suppose that $\boldsymbol{\Sigma}=0$. Then by $L_{i j k \mid l}=L_{i j l \mid k}$, we get

$$
\mu_{i \mid l} h_{j k}+\mu_{j \mid l} h_{k i}+\mu_{k \mid l} h_{i j}=\mu_{i \mid k} h_{j l}+\mu_{j \mid k} h_{l i}+\mu_{l \mid k} h_{i j} .
$$

Multiplying (27) with $y^{l}$ implies that

$$
\mu_{i}^{\prime} h_{j k}+\mu_{j}^{\prime} h_{k i}+\mu_{k}^{\prime} h_{i j}=0 .
$$

By contracting (28) with $g^{j k}$, we conclude the following

$$
(n+1) \mu_{i}^{\prime}=0 .
$$

Then on a stretch Finsler spaces, $\mu_{i}$ is constant along any geodesics. 


\section{References}

[1] Akbar-Zadeh H., Sur les espaces de Finsler à courbures sectionnelles constantes, Acad. Roy. Belg. Bull. Cl. Sci. (5) 74 (1988), no. 10, 271-322.

[2] Antonelli P.L., Handbook of Finsler geometry, Kluwer Academic Publishers, Dordrecht, 2003.

[3] Berwald L., Über Parallelübertragung in Räumen mit allgemeiner Massbestimmung, Jahresbericht D.M.V. 34 (1926), 213-220.

[4] Bácsó S., Matsumoto M., On Finsler spaces of Douglas type - a generalization of notion of Berwald space, Publ. Math. Debrecen 51 (1997), 385-406.

[5] Bácsó S., Papp I., A note on a generalized Douglas space, Period. Math. Hungar. 48 (2004), $181-184$.

[6] Chen X., Shen Z., On Douglas metrics, Publ. Math. Debrecen 66 (2005), 503-512.

[7] Ichijyō Y., Finsler manifolds modeled on a Minkowski space, J. Math. Kyoto Univ. 16 (1976), 639-652.

[8] Matsumoto M., On C-reducible Finsler spaces, Tensor (N.S.) 24 (1972), 29-37.

[9] Matsumoto M., Hōjō S., A conclusive theorem for $C$-reducible Finsler spaces, Tensor (N.S.) 32 (1978), $225-230$.

[10] Najafi B., Shen Z., Tayebi A., On a projective class of Finsler metrics, Publ. Math. Debrecen 70 (2007), $211-219$.

[11] Najafi B., Shen Z., Tayebi A., Finsler metrics of scalar flag curvature with special non-Riemannian curvature properties, Geom. Dedicata 131 (2008), 87-97.

[12] Pande H.D., Tripathi P.N., Prasad B.N., On a special form of the $h v$-curvature tensor of Berwald's connection BГ of Finsler space, Indian J. Pure. Appl. Math. 25 (1994), 1275-1280.

[13] Shen Z., Lectures on Finsler geometry, World Scientific Publishing Co., Singapore, 2001.

[14] Shen Z., Differential geometry of spray and Finsler spaces, Kluwer Academic Publishers, Dordrecht, 2001.

[15] Tayebi A., Azizpour E., Esrafilian E., On a family of connections in Finsler geometry, Publ. Math. Debrecen 72 (2008), 1-15.

[16] Tayebi A., Rafie Rad M., S-curvature of isotropic Berwald metrics, Sci. China Ser. A 51 (2008), 2198-2204. 